\title{
Protocol for Management of Peritoneal Dialysis-Related Infections in Sudan
}

\author{
Adapted from the International Society for Peritoneal Dialysis (ISPD) Peritoneal Dialysis-Related Infections Recommendations: 2005 Update * \\ Approved by the Sudan Peritoneal Dialysis Program
}

\author{
Elwaleed AM Elhassan ${ }^{\mathrm{a}^{* *}}$
}

a. MD, Sudan Peritoneal Dialysis Program

\section{Introduction}

Peritonitis is one of the major risks of peritoneal dialysis (PD), causing significant morbidity and sometimes mortality. It is also one of the principal causes of technique failure. Prevention of peritonitis is one of the keys to success with PD.

\section{Goals and targets}

- To ensure standardization of management of PD-related peritonitis between all units of the Sudan PD program (Sudan-PD).

- To achieve a peritonitis rate of no more than 1 episode every 18 months at risk ( 0.67 per year at risk) in all units of the Sudan-PD, by adopting effective preventive measures and by proper management of PD catheter exit site infections.

- To promot timely diagnosis and proper management of peritonitis in order to decrease its complications (catheter loss, technique failure and death).

\section{Recommendations}

\section{Prevention of $\mathrm{PD}$ related peritonitis}

- Prior to PD catheter insertion, take a nasal swab culture for Staph aureus, if positive, instruct the patient to apply mupirocin nasal ointment three times daily for 5 days.

- Regardless of technique, PD catheter placement must take place under strict aseptic conditions, using face masks, gowns and sterile gloves.

\footnotetext{
* Piraino B, Bailie GR, Bernardini J, Boeschoten E, Gupta A, Holmes C, Kuijper EJ, Li PK, Lye WC, Mujais S, Paterson DL, Fontan MP Ramos A, Schaefer F, Uttley L; ISPD Ad Hoc Advisory Committee. Peritoneal dialysis-related infections recommendations: 2005 update. Perit Dial Int 2005 Mar-Apr;25(2):107-31.

** Corresponding author; Division of Renal Diseases and Hypertension, University of Colorado Health Sciences Center, P.O.Box 6508, Aurora CO 80045, USA.

E mail: Elwaleed.Elhassan@UCHSC.edu
}

- Give a single dose of prophylactic antibiotic one hour before the procedure; use a first or second generation cephalosporin (e.g. cefazolin, cefuroxime).

- A two cuffed PD catheter should be used for all patients. The inner cuff must be placed within or below the rectus muscle to provide structural support for the catheter. The superficial cuff should be placed at least $2 \mathrm{~cm}$ deep to the exit site. The subcutaneous tunnel must have a downward (or if necessary lateral) direction, and the exit site must be created with a puncture hole rather than a scalpel to produce a tight fit around the catheter and avoid the use of sutures. The exit site should be placed in a location that is easily accessible to the patient for future care. It should not be placed on the belt line, on a scar or under an abdominal hang in obese patients.

- After implantation, the exit site should be covered with several layers of sterile gauze; avoid transparent and occlusive dressings because drainage tends to pool at the exit site and in the wound therefore encouraging bacterial overgrowth. Instruct the patient to keep the dressing dry and clean. Sponge bathing may be used for 2-3 weeks until the exit site is well-healed. The dressing should be changed after one week unless there is obvious bleeding or signs of infection, or sweating that makes the exit site wet, or the patient feels itchy under the taped skin, or if the stickiness of the tape is lost. Dressing changes should be restricted to trained nurses under aseptic conditions using face masks and sterile gloves.

- The double bag system and the flush before fill technique should be used for all patients. Adequate patient training reinforced by home visits is an effective tool in reducing PD-related infections.

- Aseptic technique should be practiced every time the catheter or exit site is handled. This includes proper hand washing, proper preparation of the work area, and proper handling of equipment and supplies 
- Proper hand washing refers to scrubbing the hand for at least 15-20 seconds with the six steps method, using clean tap water and antibacterial soap. If tap water is contaminated or is coming from a well, bottled or boiled water should be used instead. If liquid soap is not available, a soap bar can be used. If soap and water are not available, an alcohol-based cleansing agent that contains at least $60 \%$ isopropanol or ethanol should be used instead.

- If a cleansing agent is used for hand washing, sufficient amounts should be used to wet the hands all over, followed by scrubbing the hand with the six steps method without using a towel to dry the hands. If hands are dry in less than 15 seconds, the user has not applied enough amount of sanitizer. Cleanser type may be individualized based upon allergies, but it should not be transferred to another container because of the risk of contamination.

- Exchanges and exit site care should be performed in a clean room, with the doors and windows closed and the fans turned off. There should be no animals in the room. Supplies should be collected on a clean table.

- The tips of the transfer set and the Y-shaped giving set should not touch anything while performing the exchanges. If the end of the Y-shaped giving set is contaminated, the double bag must be discarded and a new set used instead. If the end of the transfer set is contaminated, the patient must not continue with the exchange and he/she must present to the center for transfer set changing. Prophylactic antibiotics should be prescribed for two days in case of transfer set contamination.

- If medications are to be infused into the medication port of the bag, povidone iodine should be applied to the port until it dries and the tip of the syringe should not be allowed to touch anything.

- The PD catheter and transfer set should remain securely taped to the abdomen at all times to minimize trauma. Some patients may use a belt or immobilizer. When securing the PD catheter, ensure that the catheter follows its natural direction and does not bend at its connection to the adapter, as the catheter can crack over time and this puts the patient at risk for getting peritonitis.

- Tub baths should not be taken with a PD catheter in place to avoid contamination with water borne organisms. Showers may be taken once the exit site is well healed, but the patient must avoid scrubbing the exit site. After showers, the exit site should be dried first with a clean personal towel, then the rest of the body.
- Routine exit site care should be done daily by the patient and after each shower. For cleaning the exit site care, it is preferred to use a piece of gauze saturated with normal saline, followed by the application of iodine and prophylactic gentamicin cream. Crusts should not be forcibly removed, and the exit site should never be handled with sharp instruments. If the exit site is well healed there is no need to cover it with gauze in adults. However, the use of sterile occlusive dressings is recommended for children and has been shown to result in fewer exit site infections in this group of patients.

- The abdomen should be dry and prophylactic antibiotics should be given before invasive procedure involving the abdomen or pelvis (e.g. PD catheter fluoroscopy, colonoscopy, renal transplant or endometrial biopsy) or extensive dental procedures.

- Patients should be instructed on the importance of regular bowel habits and avoidance of constipation; a high fiber diet may be helpful but laxatives (e.g. lactulose, macrogols) are often required.

- To reduce the incidence of fungal peritonitis, patients should receive prophylactic fluconazole with every antibiotic course of two weeks duration or more. Nystatin is a second line alternative to fluconazole.

\section{Management of exit site and tunnel infections}

- Exit site infection is defined by the presence of purulent drainage with or without erythema at the catheterepidermal interface. A scoring system was developed for monitoring the exit site. Infection should be assumed with an exit site score of 4 or greater; however, purulent discharge is enought by itself to diagnose exit site infection (Table 1).

- Isolated peri-catheter erythema is often a simple skin reaction to trauma, but may also be an early sign of infection. Ask the patient about accidental catheter trauma in the past few days. Advice the patient to perform exit site care twice daily using povidone iodine followed by dilute hydrogen peroxide, and to avoid occlusive dressing. Any worsening should be reported immediately, and reassessment by the PD team should be arranged within one week.

- Positive culture in the absence of abnormal appearance at the exit site is indicative of colonization rather than infection. More intensive exit site care is sufficient. 
Table 1: Exit site scoring system

\begin{tabular}{llll}
\hline & 0 point & $\mathbf{1}$ point & 2 points \\
\hline Swelling & No & $<0.5 \mathrm{~cm}$ (exit only) & $>0.5 \mathrm{~cm}$ (or tunnel) \\
Crust & No & $<0.5 \mathrm{~cm}$ & $>0.5 \mathrm{~cm}$ \\
Redness & No & $<0.5 \mathrm{~cm}$ & $>0.5 \mathrm{~cm}$ \\
Pain & No & Slight & Severe \\
Drainage & No & Serous & Purulent* \\
\hline
\end{tabular}

* purulent drainage is diagnostic of exit site infection, even if isolated.

- Tunnel infection is characterized by erythema, edema or tenderness over the subcutaneous pathway, but can be clinically occult. Tunnel infection usually occurs in the presence of an exit site infection and rarely occurs alone. During examination of the catheter exit site, the catheter tract should be milked to see if any drainage is present and observed for evidence of erythema overlying the tunnel.

- Staphylococcus aureus and Pseudomonas aeruginosa exit site infection are very often associated with concomitant tunnel infection, and these are the organisms that most often result in catheter infection related peritonitis, aggressive management is always indicated for these organisms.

- Exit site infections can be managed initially by PD nurses using appropriate protocols. If a clinical diagnosis of exit site and/or tunnel infection is made, take a swab from the drainage for Gram stain and culture. Start empiric oral therapy with amoxicillin, cephalexin, or clarithromycin unless Gram negative infection is suspected, in which case oral ciprofloxacin should be used. Avoid ciprofloxacin in children less than 12 years old and use intraperitoneal (IP) ceftazidime instead.

- The exit site should be cleaned twice daily in case of infection. If exit site infection is particularly severe, apply hypertonic saline dressings twice daily in addition to antibiotic therapy. The procedure involves adding 1 table spoon of salt to $500 \mathrm{ml}$ of sterile water, applying this solution to gauze and wrapping it around the exit site for 15 minutes.

- Modify treatment according to culture results. Treatment should continue for at least seven days after resolution of all signs of infection, with a minimum total duration of two weeks. Treatment with two antibiotics, including ciprofloxacin, is indicated for Pseudomonas aeruginosa infections, with the addition of IP ceftazidime if the infection resolves slowly or recurs. Removal of the catheter is indicated if fungal infection is confirmed to prevent progression to fungal peritonitis.
- If the infection persists after two weeks of antibiotic therapy:

- Ascertain that the patient is complying with the therapeutic regimen.

- Re-examine the catheter for evidence of tunnel infection. An ultrasound scan should be performed to exclude a small abscess formation along the tunnel tract.

- An exposed external cuff should be completely shaved off under local anesthetic with debridement of the surrounding tissue to help clear the infection.

- Another two weeks of antibiotic therapy may be attempted provided that infection has not progressed, no abscess is present, and any exposed cuff has been trimmed.

- The catheter should be removed if the infection has progressed despite two weeks of antibiotic therapy, an abscess is present, fungal infection has been confirmed, or peritonitis has developed.

- Catheter removal should be via surgical dissection rather than traction to avoid retention of the cuffs. Antibiotics should be continued for one to two weeks after catheter removal. If the patient does not have peritonitis, a new catheter can be placed simultaneously in the opposite lower quadrant. PD may be resumed immediately, initially in the supine position.

\section{Diagnosis of PD related peritonitis}

- Assume peritonitis in all patients presenting with cloudy effluent, and attempt to confirm it by obtaining effluent cell count, differential, and culture. Consider the possibility of peritonitis in any PD patient presenting with abdominal pain, fever, nausea or general ill health even if the effluent is clear.

- To prevent delay in treatment, peritonitis can be managed initially by PD nurses using appropriate protocols. 
- Start antibiotic therapy as soon as cloudy effluent is seen and a sample of the effluent has been taken, without waiting for confirmation by the cell count from the laboratory. In a suspected case of peritonitis, carefully inspect the exit site and the tunnel of the catheter for evidence of catheter infection; culture any drainage from the exit site along with the effluent.

- Patients who reside in remote locations should report symptoms suggestive of peritonitis immediately to the center, keep the cloudy effluent bag until they are able to bring in the sample for analysis, and possibly initiate IP antibiotics at home.

- Send the whole bag to the microbiology department (preferably the first bag noticed to contain a cloudy effluent) and request effluent cell count and differential, gram stain, and culture. Effluent white blood cells (WBC) more than $100 / \mu \mathrm{L}$, with at least $50 \%$ polymorphonuclear neutrophil cells, confirm the diagnosis of peritonitis. Gram stain is important in that it may indicate the presence of fungi, thus allowing for prompt initiation of antifungal therapy and permitting timely arrangement of catheter removal. With this exception, empiric therapy should not be based on the Gram stain, but should follow the protocol as outlined below. A positive culture in the absence of a significant WBC count represents contaminants and should not be considered as peritonitis.

- To attain the highest possible culture yield, centrifuge 50 $\mathrm{ml}$ of peritoneal effluent at $3000 \mathrm{~g}$ for 15 minutes, mix the sediment in $3-5 \mathrm{ml}$ of sterile saline and inoculate this material both on solid culture media (incubated in aerobic, micro-aerophilic, and anaerobic environments) and into a standard blood culture medium. If equipment for centrifuging large amounts of fluid is not available; inject $5-10 \mathrm{ml}$ of effluent directly into blood culture bottles. If blood culture bottles and centrifugation equipment are not available; shake the bag manually, take $5 \mathrm{ml}$ of effluent and inoculate in solid culture media. Store the whole bag in an incubator to encourage growth and test the effluent daily if feasible.

\section{Treatment principles and steps}

- Relieve pain using paracetamol and opioid analgesics (e.g. tramadol, morphine, pethidine) for patients with significant residual renal function (RRF). Use NSAIDs for patients without significant RRF (e.g. Ibuprofen, diclofenac).
- Start empirical IP treatment with a first and third generation cephalosporin (e.g. cefazolin plus ceftazidime). Aminoglycosides can be used as alternatives to third generation cephalosporins for gram negative coverage if third generation cephalosporins are not available.

- Aminoglycoside use carries the risk of irreversible cochlear and vestibular ototoxicity especially with large doses, prolonged or frequent administration and increasing age. To reduce this risk, use intermittent IP dosing.

- The above regimen is recommended for pediatric patients unless the child has fever, severe abdominal pain, or a risk factor for severe infection (e.g. history of MRSA infection, recent or current evidence of PD catheter infection, exit site or nasal colonization with Staphylococcus aureus, or age less than 2 years). In such cases use vancomycin or teicoplanin in combination with a third generation cephalosporin as initial therapy

- Patients with extremely cloudy effluent may benefit from heparinization of the dialysate (500 units of heparin per liter) to prevent occlusion of the catheter by fibrin. Refrain from using concentrated fluids during episodes of peritonitis.

- Within 48 hours of initiating therapy, most patients will show considerable clinical improvement and the effluent will be visibly clearing. By this time the initial culture results should have come allowing treatment to be modified accordingly. If there is no improvement after 48 hours, consider placing the patient on IPD for 24 hours in an attempt to clear the effluent. Then, keep the abdomen dry for 2-3 days apart from a single daily exchange with antibiotics. There is some evidence that white cells in peritoneum function better in the absence of any dialysate.

- After 72 hours of initiating therapy, repeat peritoneal effluent WBC count and differential. If the infection is resolving, continue treatment for two weeks and change the transfer set after completion of therapy. If the effluent WBC count reveals that the infection is not resolving and the first culture result remains negative at 72 hours, send another effluent sample for culture and consider the possibility of fungal or mycobacterial peritonitis. Continue with the initial antibiotic regimen for another two days.

- Assess the patient at day five, if he/she has improved, leave the catheter in place. Modify the antibiotics according to culture results or continue with the initial regimen if the second culture is also negative. Continue treatment for at least two weeks and change the transfer set after completion of therapy. 
Table 2: Terminology of peritonitis

\begin{tabular}{ll}
\hline Recurrent peritonitis & $\begin{array}{l}\text { An episode that occurs within } 4 \text { weeks of completion of therapy of a prior episode but with a different } \\
\text { organism }\end{array}$ \\
Relapsing peritonitis & $\begin{array}{l}\text { An episode that occurs within } 4 \text { weeks of completion of therapy of a prior episode with the same } \\
\text { organism or one sterile episode }\end{array}$ \\
Repeat peritonitis & $\begin{array}{l}\text { An episode that occurs more than } 4 \text { weeks after completion of therapy of a prior episode with the } \\
\text { same organism }\end{array}$ \\
Refractory peritonitis & Failure of the effluent to clear after 5 days of appropriate antibiotics therapy \\
Catheter related peritonits & $\begin{array}{l}\text { Peritonitis in conjunction with an exit-site or tunnel infection with the same organism or one site } \\
\text { sterile }\end{array}$
\end{tabular}
sterile

- If the patient has not improved by day five and the second culture is positive, modify the antibiotics according to the second culture results. Assess the patient after another five days and remove the catheter if there is still no clinical improvement.

- If the patient has not improved by day five and the second culture is also negative, remove the catheter and change the antibiotic regimen to vancomycin plus gentamicin. Continue treatment for at least two weeks.

- Total duration of therapy should be at least two weeks. Peritonitis due to Staphylococcus aureus, Gram negative organisms, or enterococci should be treated for a minimum duration of 3 weeks whether the catheter was removed or not.

- Peritonitis due to Pseudomonas aeruginosa should always be treated with a combination of two antibiotics.

- Peritonitis due to fungal organisms (diagnosed by microscopy or culture) mandates immediate catheter removal preceded by peritoneal lavage until the effluent is clear to avoid the formation of adhesion and lower the fungal burden.

- Fluconazole for four weeks (initially IP), is the drug of choice for fungal peritonitis. For patients with severe infections and those who have had significant prior exposure to azole antifungals, add amphotericin B (test dose required) until the culture results are available with susceptibilities.

- Mycobacterial peritonitis should be suspected in any patient with prolonged failure to thrive, prolonged symptoms despite antibiotic therapy, or relapsing peritonitis with negative bacterial cultures.

\section{Indications for catheter removal}

- When indicated, early catheter removal is crucial to reduce the mortality and morbidity. The primary goal should always be optimal patient treatment and protection of the peritoneum, and not saving the catheter. Prolonged attempts to treat refractory peritonitis are associated with extended hospital stay, peritoneal membrane damage, and, in some cases, death. Death related to peritonitis, defined as death of a patient with active peritonitis, or admitted with peritonitis, or within 2 weeks of a peritonitis episode, should be a very infrequent event.

- The catheter should be removed in the following conditions: fungal peritonitis, refractory peritonitis, relapsing peritonitis, catheter related peritonitis, peritonitis related to an intra-abdominal pathology, and refractory exit site or tunnel infection (Table 2).

- If the catheter is removed because of relapsing peritonitis and the effluent has rapidly cleared, simultaneous catheter placement can be attempted. In case of refractory or fungal peritonitis, a minimum period of 4 weeks is recommended between the time of catheter removal and placement of a new catheter.

- It is preferred to re-insert PD catheters surgically or laparoscopically following an episode of peritonitis because of the risk of adhesions. The catheter should also be sutured into the pelvis at the first attempt of reinsertion.

\section{Preventing recurrence of peritonitis}

- Every episode of peritonitis should be perceived as a potentially preventable event and thoroughly investigated by the PD team. 
- Enquire from the patient carefully and in a nonthreatening manner about the possible mechanism of the episode (e.g. poor aseptic technique, lack of clean area to carry out exchanges, poor eyesight, recent exit-site infection, constipation or diarrhea) and advice accordingly.

- Re-train patients and arrange for a home visit after each episode of peritonitis, home visits are invaluable in detecting errors in the technique.

- Following each episode of peritonitis, fill out the Sudan-PD unified peritonitis questionnaire.

\section{Drug delivery}

- With strict adherence to aseptic technique, IP administration of antibiotics and antifungal agents is preferred to IV administration. IP antibiotics can be given in each exchange (continuous dosing) or once daily (intermittent dosing).
- Continuous dosing is preferred for inpatient treatment, while intermittent dosing is preferred for outpatient treatment (Tables 3 and 4).

- In intermittent dosing, the antibiotic-containing dialysis solution must be allowed to dwell for at least 6 hours (utilizing the night exchange) to allow adequate absorption of the antibiotic into the systemic circulation.

- Vancomycin, aminoglycosides and cephalosporins can be mixed in the same dialysis solution bag without loss of bioactivity. However, aminoglycosides should not be added to the same exchange with penicillins because of chemical incompatibility.

- For any antibiotics that are to be mixed, separate syringes must be used for adding the antibiotics. Some antibiotics are incompatible if combined in the same syringe but compatible when mixed in dialysis solutions, e.g. vancomycin and ceftazidime (1 liter or higher). 
Table 3: Recommendations for IP antibiotic dosing for anuric adult CAPD patients (increase dose by $25 \%$ for patients producing more than $100 \mathrm{ml}$ of urine daily)

\begin{tabular}{|c|c|c|}
\hline Generic name & $\begin{array}{l}\text { Intermittent } \\
\text { (one exchange per day) }\end{array}$ & $\begin{array}{l}\text { Continuous } \\
\text { (all exchanges, mg per liter) }\end{array}$ \\
\hline Amikacin & $2 \mathrm{mg} / \mathrm{kg}$ & LD 25, MD 12 \\
\hline Amoxicillin & ND & LD 250-500, MD 50 \\
\hline Amphotericin & ND & MD 1.5 \\
\hline Ampicillin & ND & MD 125 \\
\hline Ampicillin/sulbactam & $2 \mathrm{~g}$ every 12 hours & LD 1000, MD 100 \\
\hline Aztreonam & ND & LD 1000, MD 250 \\
\hline Azlocillin & ND & LD 500, MD 250 \\
\hline Cefazolin & $15 \mathrm{mg} / \mathrm{kg}$ & LD 500, MD 125 \\
\hline Ceftazidime & $1-1.5 \mathrm{~g}$ & LD 500, MD 125 \\
\hline Ceftizoxime & $1 \mathrm{~g}$ & LD 250, MD 125 \\
\hline Cephalothin & $15 \mathrm{mg} / \mathrm{kg}$ & LD 500, MD 125 \\
\hline Cephradine & $15 \mathrm{mg} / \mathrm{kg}$ & LD 500, MD 125 \\
\hline Cifepime & $1 \mathrm{~g}$ & LD 500, MD 125 \\
\hline Ciprofloxacin & ND & LD 50, MD 25 \\
\hline Clindamycin & ND & LD 300, MD 125 \\
\hline Fluconazole & $200 \mathrm{mg}$ & NA \\
\hline Gentamicin & $0.6 \mathrm{mg} / \mathrm{kg}^{*}$ & LD 8, MD 4 \\
\hline Imipenem/cilastatin & $1 \mathrm{~g} \mathrm{bd}$ & LD 500, MD 200 \\
\hline Nafcillin & ND & MD 125 \\
\hline Netilmicin & $0.6 \mathrm{mg} / \mathrm{kg}$ & LD 8, MD 4 \\
\hline Oxacillin & ND & MD 125 \\
\hline Penicillin G & ND & LD 50000, MD 25000 units \\
\hline Teicoplanin & $15 \mathrm{mg} / \mathrm{kg}$ & $\mathrm{LD} 400, \mathrm{MD} 40 * *$ \\
\hline Tobramycin & $0.6 \mathrm{mg} / \mathrm{kg}$ & LD 8, MD 4 \\
\hline Vancomycin & $30 \mathrm{mg} / \mathrm{kg}$ every $5-7$ days $* * *$ & LD 1000, MD 25 \\
\hline
\end{tabular}

* Check blood level every 3-4 days (target 2-4 mg/l), if level $<2 \mathrm{mg} / 1$ increase dose by $0.2 \mathrm{mg} / \mathrm{kg}$, if level $>4 \mathrm{mg} / 1 \mathrm{reduce}$ dose by $0.2 \mathrm{mg} / \mathrm{kg}$, if level $>7 \mathrm{mg} / \mathrm{l}$ miss a day and reduce dose by $0.2 \mathrm{mg} / \mathrm{kg}$.

** In each bag for 7 days, then in 2 bags/day for 7 days, then in 1 bag/day for 7 days.

*** Check blood level on day 4 (target $>10 \mathrm{mg} / \mathrm{l}$ ), if level $\leqslant 12 \mathrm{mg} / \mathrm{l}$ repeat dose every 5 days, if level 13-14 mg/l repeat dose every 6 days, if level $\geq 15 \mathrm{mg} / \mathrm{l}$ repeat dose every 7 days (usual dose for anuric patients).

LD: loading dose in mg, MD: maintenance dose, ND: no data, NA: not applicable 
Elhassan EAM

Table 4: Recommendations for IP antibiotic dosing for adult APD patients

\begin{tabular}{ll}
\hline Generic name & Intermittent dosing for APD patients (long dwell) \\
\hline Cefazolin & $20 \mathrm{mg} / \mathrm{kg}$ once daily \\
Cifepime & $1 \mathrm{~g}$ once daily \\
Fluconazole & $200 \mathrm{mg}$ once daily every $24-48 \mathrm{hours}$ \\
Tobramycin & $1.5 \mathrm{mg} / \mathrm{kg}$ loading dose then $0.5 \mathrm{mg} / \mathrm{kg}$ once daily \\
Vancomycin & $30 \mathrm{mg} / \mathrm{kg}$ loading dose then $15 \mathrm{mg} / \mathrm{kg}$ once daily every $3-5$ days \\
\hline
\end{tabular}




\section{Algorithm 1 \\ Management of Exit Site Infection}

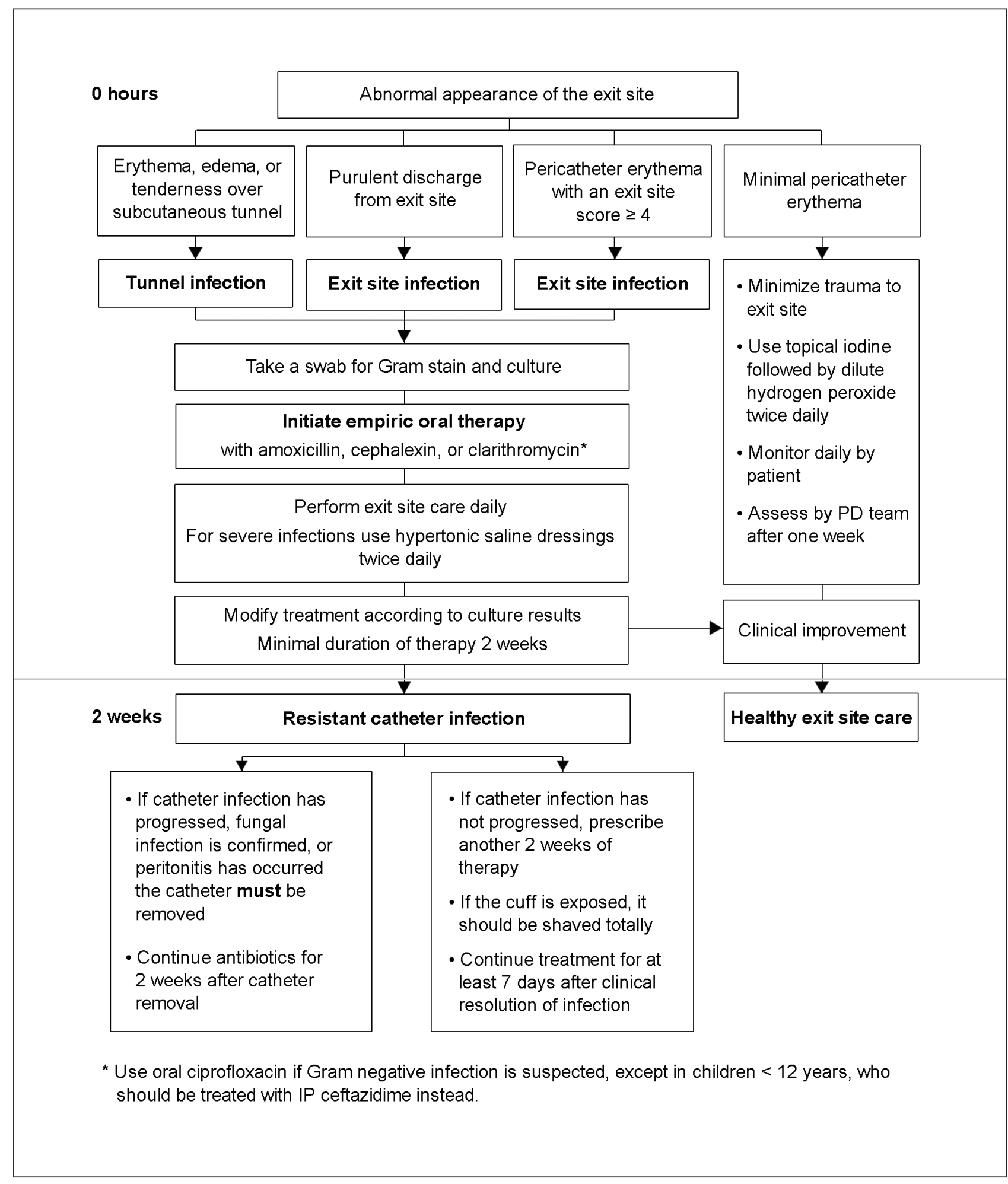




\section{Algorithm 2 \\ Management of PD Related Peritonitis}

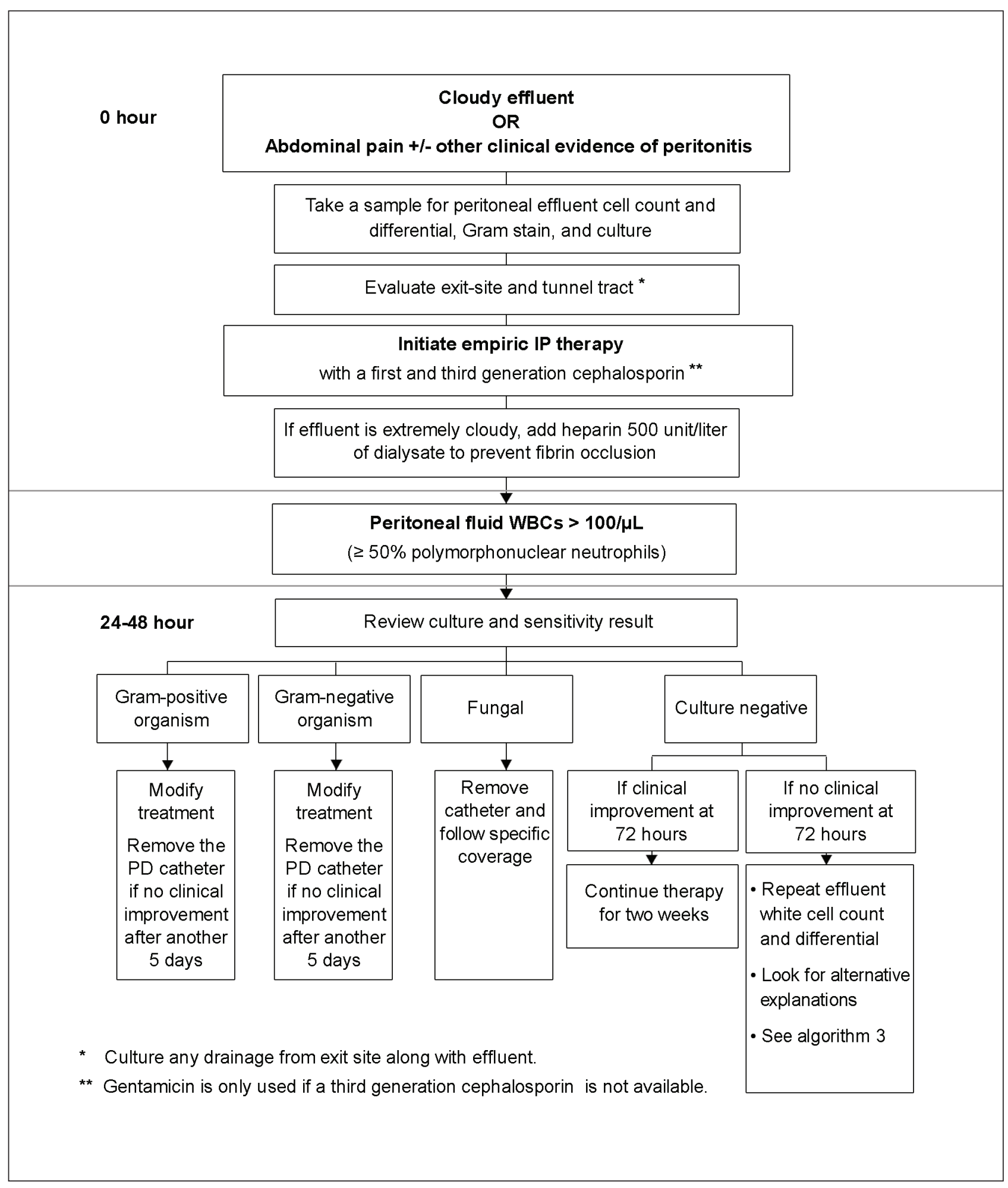


Algorithm 3

Management of PD Related Culture Negative Peritonitis

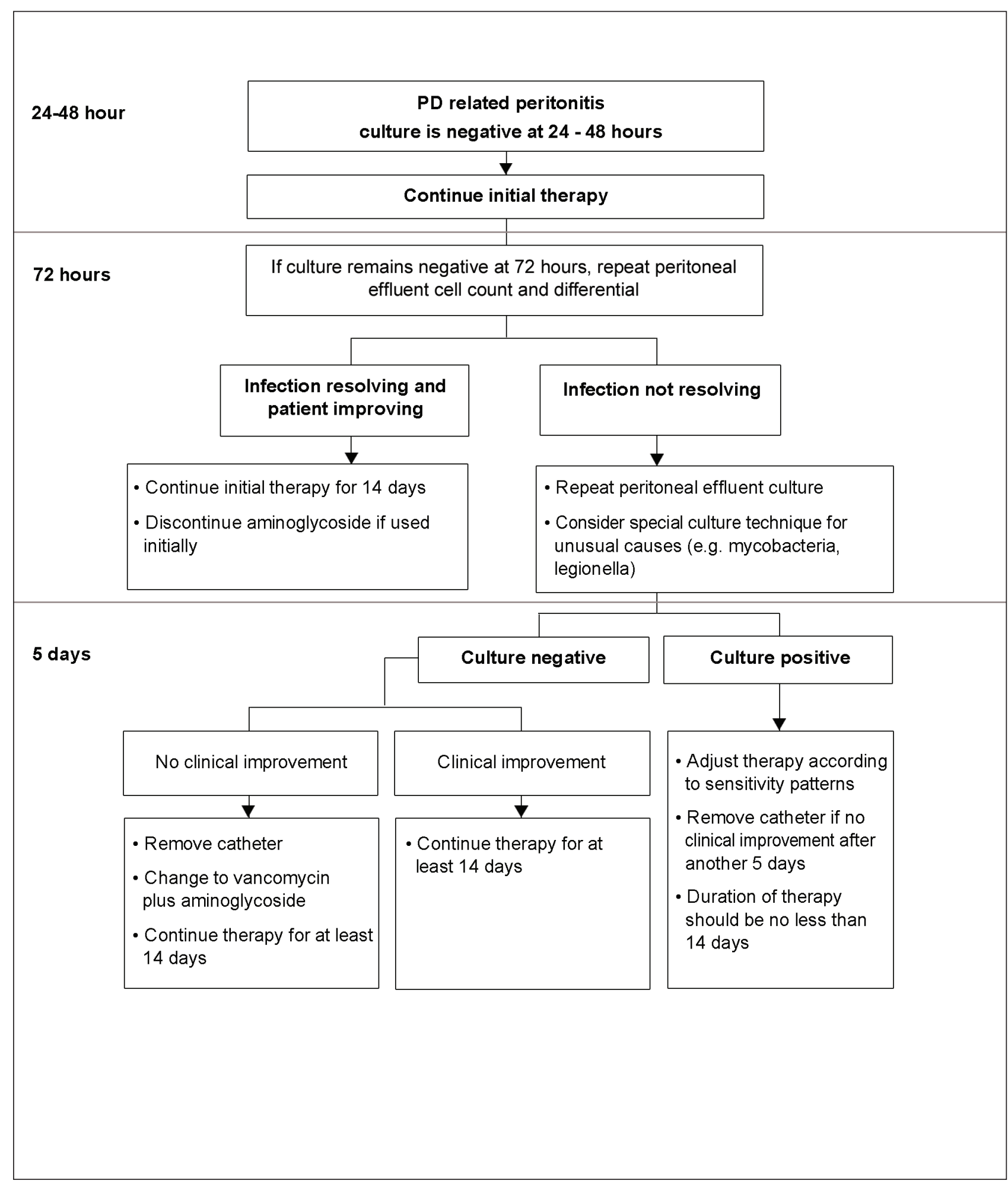




\section{Algorithm 4 \\ Management of Fungal Peritonitis}

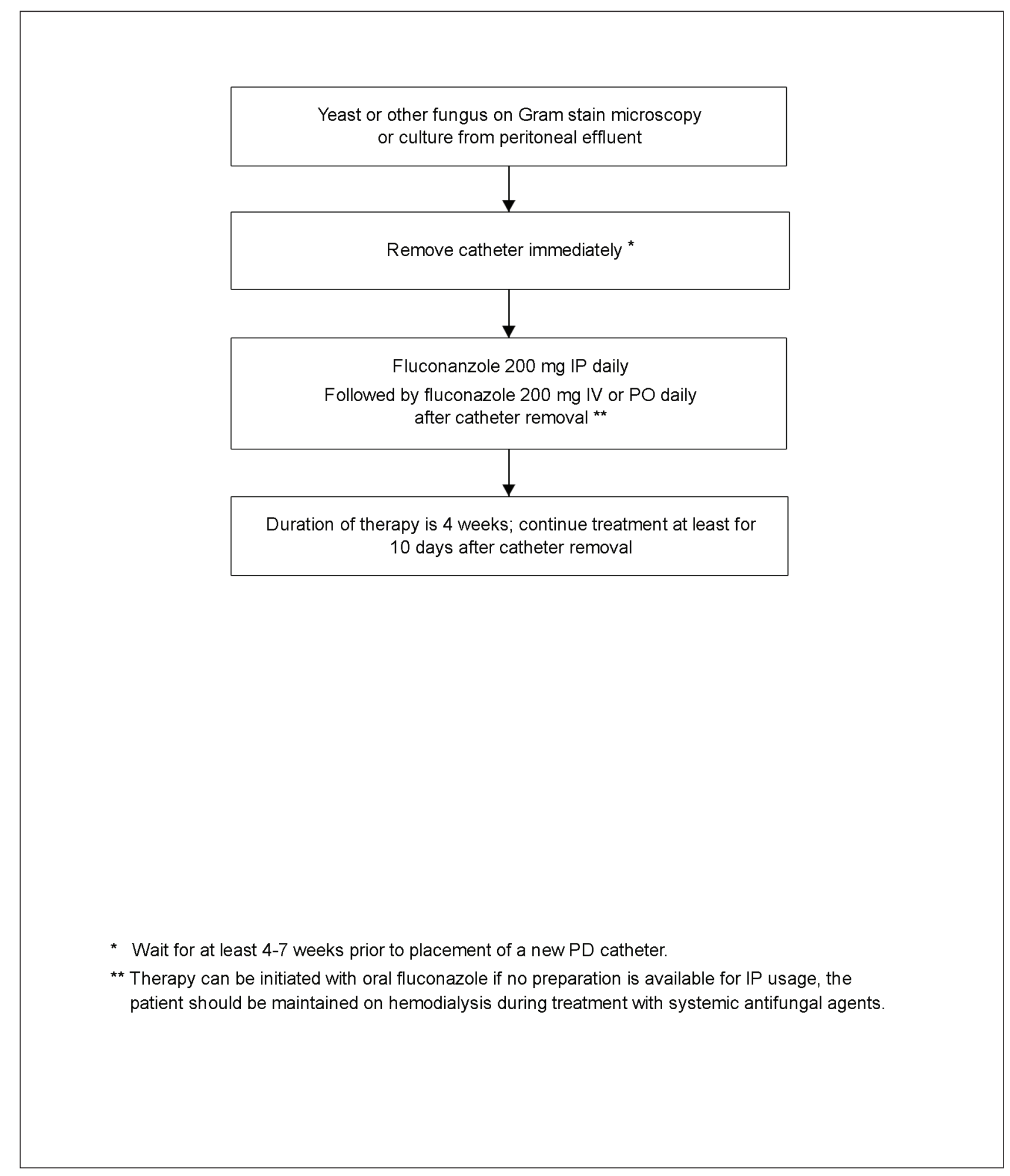

